\title{
Stochastic model of optical variability of BL Lacertae
}

\author{
D. A. Blinov ${ }^{1,2}$ and V. A. Hagen-Thorn ${ }^{1,2}$ \\ 1 St.-Petersburg State Univ., Universitetsky pr. 28, 198504 St.-Petersburg, Russia \\ e-mail: dmitriy.blinov@gmail.com \\ 2 Isaac Newton Institute of Chile, St.-Petersburg Branch, Russia
}

Received 10 June 2009 / Accepted 20 June 2009

\section{ABSTRACT}

\begin{abstract}
Context. We use optical photometric and polarimetric data of BL Lacertae that cover a period of 22 years to study the variability of the source.

Aims. The long-term observations are employed for establishing parameters of a stochastic model consisting of the radiation from a steady polarized source and a number of variable components with different polarization parameters, proposed by the authors early. Methods. We infer parameters of the model from the observations using numerical simulations based on a Monte Carlo method, with values of each model parameter selected from a Gaussian distribution. We determine the best set of model parameters by comparing model distributions to the observational ones using the $\chi^{2}$ criterion.

Results. We show that the observed photometric and polarimetric variability can be explained within a model with a steady source of high polarization, $\sim 40 \%$, and with direction of polarization parallel to the parsec scale jet, along with $10 \pm 5$ sources of variable polarization.
\end{abstract}

Key words. galaxies: active - methods: statistical - galaxies: BL Lacertae objects: individual: BL Lac

\section{Introduction}

BL Lacertae is an active galactic nucleus $(z=0.069)$ that is the prototype of a class of AGN, BL Lac's objects, which are characterized by strong dominance of non-thermal continuum, extreme optical variability, and high optical polarization (Angel \& Stockman 1980). BL Lac objects belong also to the class of blazars with highly variable emission from radio wavelengths to $\gamma$-rays and superluminal apparent velocities in radio jets. Recent theoretical and observational results (e.g., Vlahakis \& Königl 2004; McKinney 2006; Marscher et al. 2008) suggest that the jets are magnetically dominated, with a helical structure of the magnetic field close to the central engine and accretion disk where the jet formation and acceleration take place. However, at some distance from the central engine the jets become kinetically dominated, with a turbulent magnetic field that is subject to ordering by shocks. If the non-thermal optical emission in blazars originates close to the millimeter-wave VLBI core, as suggested by Lister \& Smith 2000; Gabuzda et al. 2006; Jorstad et al. 2007, then the optical polarization behavior should reflect the dynamics of the magnetic field in this region.

On long-term timescales, many BL Lac objects show a preferential direction of optical polarization (Hagen-Thorn 1980, Hagen-Thorn et al. 2002 - Paper I) closely aligned with the direction of the parsec scale jet (Jorstad et al. 2007), as well as some correlations between total intensity and polarization parameters (Paper I). The data on OJ 287 (Hagen-Thorn 1980) and BL Lac (Paper I) permit to suggest a phenomenological model of variability for BL Lac object: there is a constantly acting source of polarized radiation responsible for the preferential direction of observed polarization and a number of sources with randomly distributed polarization parameters and intensities that are superimposed on the steady source.
In this paper we use simultaneous photometric and polarimetric measurements of BL Lac obtained over 22 years (for details see Paper I) and develop a method to derive the optimal number of the superimposed sources as well as the properties of the steady and superimposed sources that give the best fit to the data. We apply the results to describe the magnetic field structure in the optical emission region.

We recall that the relative Stokes parameters $p_{x}$ and $p_{y}$ are directly measured from polarimetric observations, and they are used to derive the degree of polarization $P=\left(p_{x}^{2}+p_{y}^{2}\right)^{1 / 2}$ and position angle of polarization $\Theta_{0}=0.5 \arctan \left(p_{y} / p_{x}\right)$. Photometric measurements were performed independently and were corrected for contribution of the host galaxy. The transformation from magnitudes $m$ to intensities $I$ was performed with the absolute calibration of Mead et al. (1990). Polarimetric and photometric measurements obtained on the same night were used to compute the absolute Stokes parameters $I, Q=p_{x} I$, and $U=p_{y} I$.

\section{Stochastic model}

Figures 1-6 (left column) show the observed dependences of polarization parameters on Stokes parameter I. Figure 7a presents the plot between the degree of polarization and position angle of polarization, while Fig. 8a gives the dependence between the relative Stokes parameters.

The aim of this work is to reproduce all dependences obtained from the observations with a model consisting of a steady source of polarized radiation with parameters $I^{c}, p_{x}^{c}, p_{y}^{c}$ and $n$ variable sources with parameters $I_{i}^{v}, p_{x i}^{v}, p_{y i}^{v}$, where $i=1, \ldots, n$. At a given date the observed optical emission is represented by Stokes parameters $I_{\text {mod }}, Q_{\text {mod }}$, and $U_{\text {mod }}$ that are computed as the superposition of the constantly acting and variable sources (see 

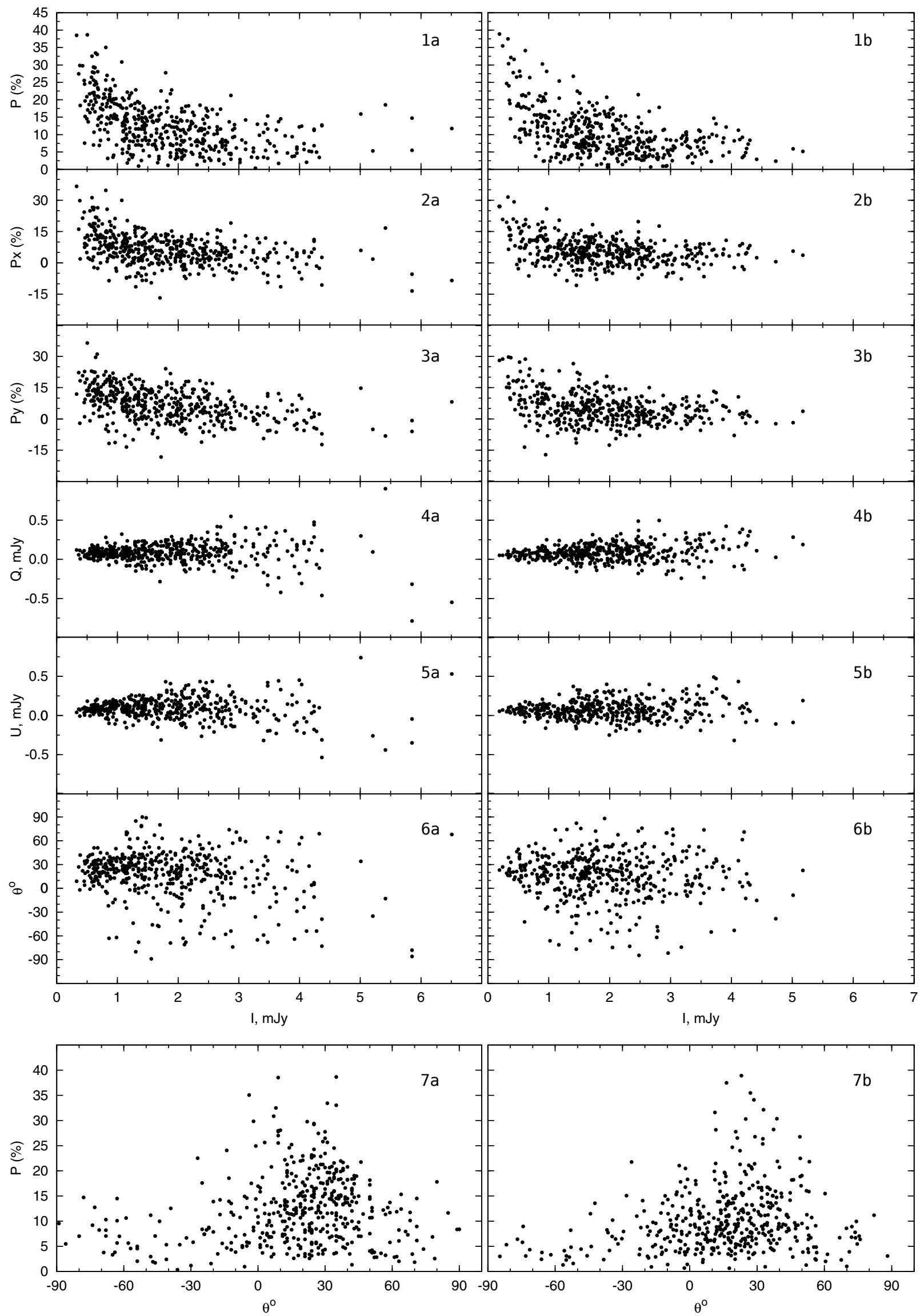

Figs. 1-7. Observed dependences (left column) and modeling results (right column) between polarization parameters and intensity. 

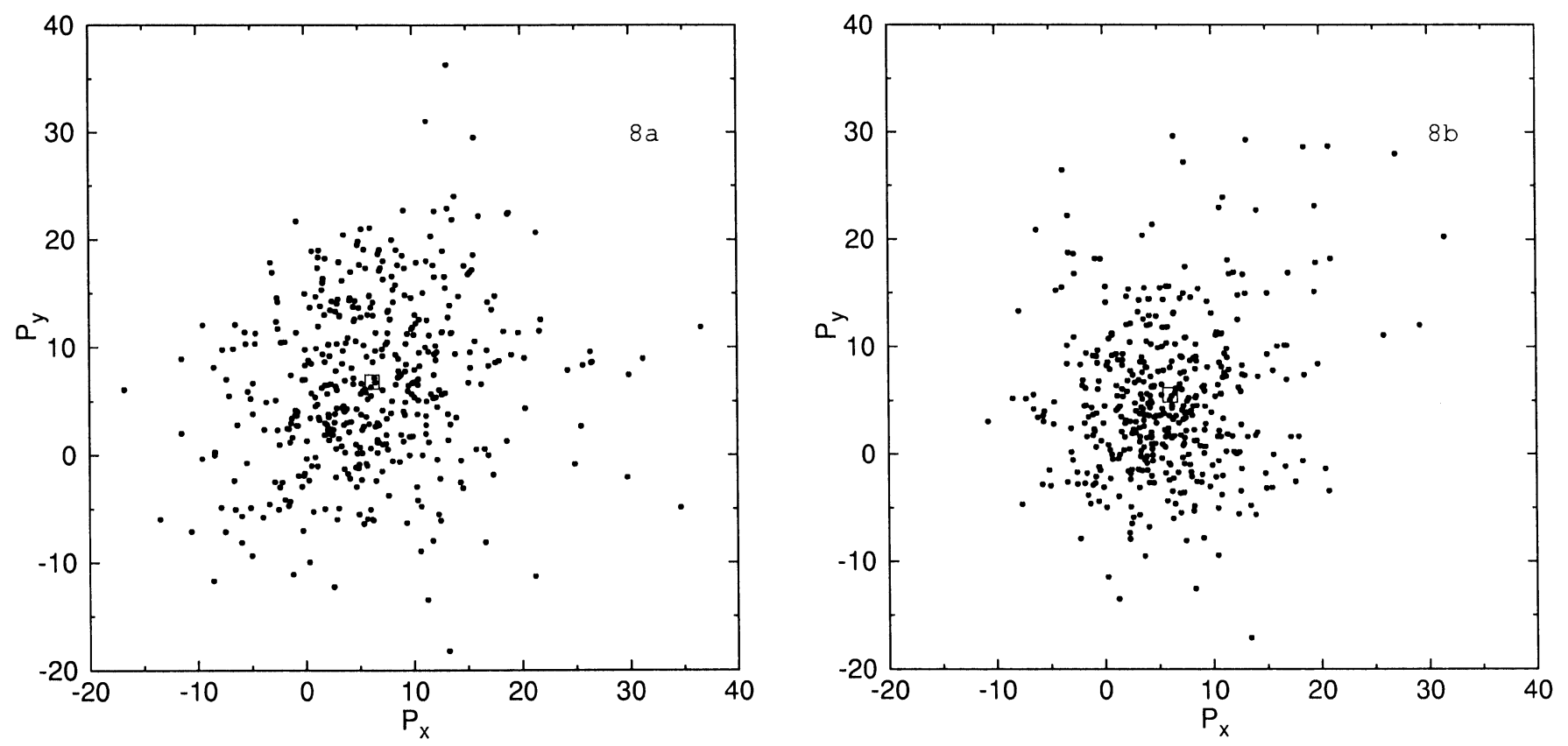

Fig. 8. Relative Stokes parameters: observed (left) and modeled (right). Mean values are indicated by open squares.

Appendix for the basic equations). The total number of dates is 451 .

All parameters are assumed to be normally distributed around their mean value (mathematical expectation $M$ ) with standard deviation $\sigma$. There are three constraints on the parameters of the steady source: 1) its flux must be less than the minimum observed flux; 2) its polarization direction must yield the preferred direction of polarization, which is $\Theta_{0}^{\text {pref }}=24^{\circ}$; and 3 ) its degree of polarization must be sufficiently high to ensure a high observed polarization al low flux levels.

Determination of optimal model parameters was accomplished by a Monte Carlo method. After multiple realizations of the model with different sets of parameters, we accept a set of parameters that produces the best agreement with the observed dependences as the most probable model. The initial criterion for rejecting obviously inadequate models and outlining possible sets of parameters was the visual similarity between the modeled and observed correlations.

The details of modelling were as follows: 1) the space of Stokes parameters $\{I, Q, U\}$ was separated into $K$ non-intersecting regions, each containing about 10 points from the observed set of 451 points; 2) from possible sets of parameters a realization of values $M(n), \sigma(n)$, $M\left(I^{v}\right), \sigma\left(I^{v}\right), M\left(p_{x}^{v}\right), \sigma\left(p_{x}^{v}\right), M\left(p_{y}^{v}\right), \sigma\left(p_{y}^{v}\right), I^{c}, p_{x}^{c}, p_{y}^{c}$ was selected by chance (the value of $p_{y}^{c}$ was calculated from the condition $\Theta_{0}^{c}=24^{\circ}$, and it was assumed that $\left.\sigma\left(p_{y}^{c}\right)=\sigma\left(p_{x}^{c}\right)\right)$; 3) using this realization we found by random chance the values $n, I^{v}, p_{x}^{v}, p_{y}^{v}, I^{c}, p_{x}^{c}, p_{y}^{c}$ for 451 cases; 4) using Eqs. (A.1)-(A.7) for each of the 451 cases, we determined $I_{\text {mod }}, Q_{\text {mod }}, U_{\text {mod }}$; and $5)$ we compared the number of points in each of $K$ regions derived in the model, $\operatorname{MOD}_{i}(i=1, \ldots, K)$, with the number of observed points, $\operatorname{OBS}_{i}(i=1, \ldots, K)$, by calculating the value of $\chi^{2}$ :

$\chi^{2}=\sum_{i=1}^{K} \frac{\left(\mathrm{MOD}_{i}-\mathrm{OBS}_{i}\right)^{2}}{\mathrm{OBS}_{i}}$
After repeating the third step 1000 times, we have found $\overline{\chi^{2}}$ for the realization chosen in the second step. Regarding $\overline{\chi^{2}}$ as a function of parameters of the second step, we have determined the set of parameters that gives a global minimum of $\overline{\chi^{2}}$ by the genetic algorithm of optimization (Goldberg 1989). We used the PyGene library to implement the algorithm. Beginning with the second step, the calculations were performed 200 times. The whole algorithm was repeated for 3 different separations of $\{I, Q, U\}$-space. This gives 600 values of parameters. The mean values that give the most probable realization are as follows: $M(n)=10 \pm 1, \sigma(n)=5 \pm 1, M\left(I^{v}\right)=0.12 \pm 0.04, \sigma\left(I^{v}\right)=$ $0.15 \pm 0.05, M\left(p_{x}^{v}\right)=2 \pm 1, \sigma\left(p_{x}^{v}\right)=16 \pm 3, M\left(p_{y}^{v}\right)=2 \pm 1, \sigma\left(p_{y}^{v}\right)=$ $18 \pm 3, I^{c}=0.19 \pm 0.03, p_{x}^{c}=27 \pm 5, p_{y}^{c}=28 \pm 4$.

\section{Results and conclusions}

We have constructed the dependences between the polarization parameters and intensity similar to the observed dependences using such set of parameters: $M(n)=10, \sigma(n)=5, M\left(I^{v}\right)=$ $0.12, \sigma\left(I^{v}\right)=0.15, M\left(p_{x}^{v}\right)=2, \sigma\left(p_{x}^{v}\right)=16, M\left(p_{y}^{v}\right)=2, \sigma\left(p_{y}^{v}\right)=$ $18, I^{c}=0.19, p_{x}^{c}=27, p_{y}^{c}=28$. Figures $1 \mathrm{~b}-8 \mathrm{~b}$ give the modeled dependences obtained for one realization of parameters taken at random from this set. There is an excellent agreement between the observed and modelled dependences. The similarity is excellent for other realizations of this set of parameters as well. Figures 9 and 10, as examples, give two dependences obtained for two more different realizations. In addition, since the value of $\sigma\left(I^{v}\right)$ is higher than $M\left(I^{v}\right)$ in some cases we formally may obtain by chance a negative value of the intensity $I^{v}$; we exclude such cases.

The proposed stochastic model agrees very well with the phenomenological model suggested in Paper I. The model gives a high degree of polarization for the steady source, $P \sim 40 \%$, with direction of polarization along the jet. This can be produced by a toroidal component of a helical magnetic field in the magnetically dominated part of the jet. Such a scenario implies that the optical emission most likely comes from the acceleration 


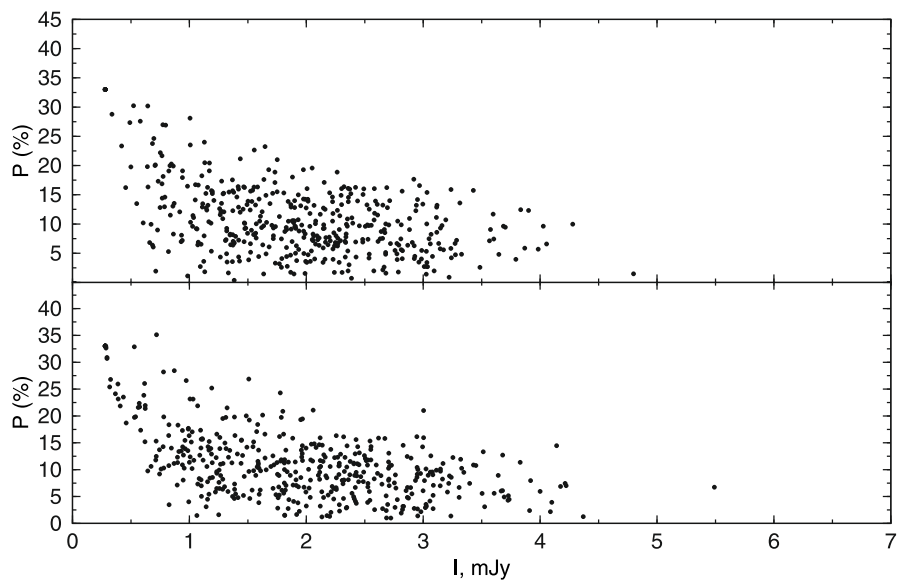

Fig. 9. The dependence of the degree of polarization on flux for two different realizations.

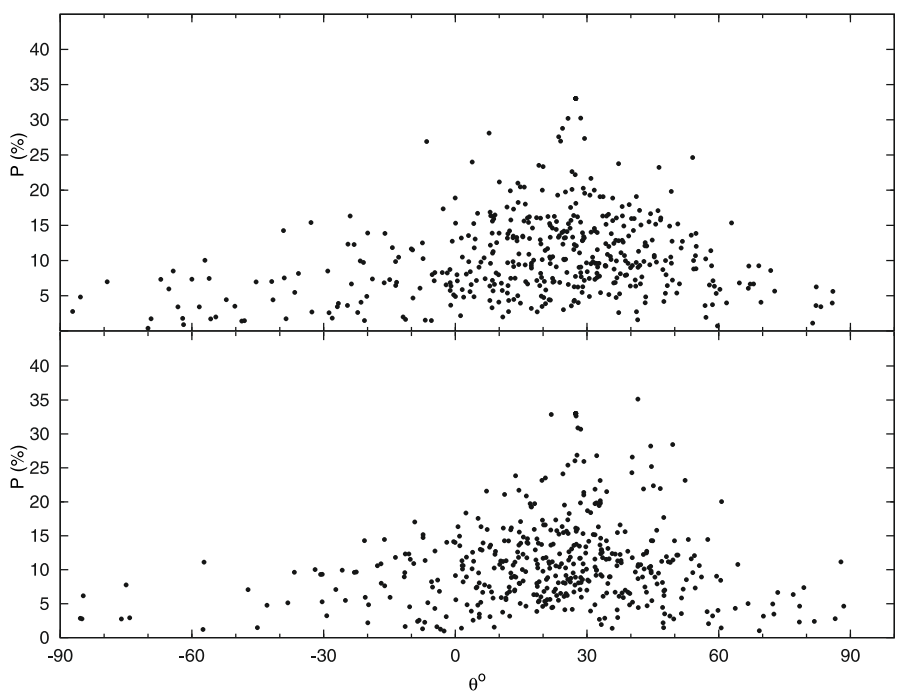

Fig. 10. The dependence of the degree of polarization on its direction for two different realizations.

zone of the jet, as suggested by Marscher et al. (2008), who recently have observed in BL Lac steady rotation of the position angle of optical polarization by about $240^{\circ}$ over a five-day interval. The rotation can be explained within a helical field structure located in the acceleration zone $\sim 0.4 \mathrm{pc}$ upstream of the $\mathrm{mm}$ wave VLBI core. However, the intensity of the steady source is only $\sim 50 \%$ higher than the most probable intensity of a random variable source, while at a given moment we expect $\sim 10$ such sources to operate simultaneously. The most probable polarization of variable sources $\sim 3 \%$, which implies that each variable source is an emission region with a significant number of cells with randomly oriented magnetic field. This means that, in general, the magnetic field is dominated by a turbulent component that masks the toroidal component. However, $\sigma p_{x}^{v}$ and $\sigma p_{y}^{v}$ are fairly high, which requires ordering of the magnetic field in the emission regions of the variable sources at some times. The latter can be connected with propagation of disturbances along the jet and formation of shocks in the optical emission region.

Acknowledgements. The work was supported by RFBR grant 09-02-00092.

\section{Appendix}

Because of additive nature of absolute Stokes parameters, the main equations for obtaining the model values are as follows:

$$
\begin{aligned}
& I_{\text {mod }}=I^{c}+\sum_{i=1}^{n} I_{i}^{v}, \\
& Q_{\text {mod }}=p_{x}^{c} I^{c}+\sum_{i=1}^{n} p_{x i}^{v} I_{i}^{v}, \\
& U_{\text {mod }}=p_{y}^{c} I^{c}+\sum_{i=1}^{n} p_{y i}^{v} I_{i}^{v} .
\end{aligned}
$$

From (A.1)-(A.3) for relative Stokes parameters we have

$$
\begin{gathered}
p_{x \bmod }=\frac{p_{x}^{c} I^{c}+\sum_{i=1}^{n} p_{x i}^{v} I_{i}^{v}}{I^{c}+\sum_{i=1}^{n} I_{i}^{v}}, \\
p_{y \text { mod }}=\frac{p_{y}^{c} I^{c}+\sum_{i=1}^{n} p_{y i}^{v} I_{i}^{v}}{I^{c}+\sum_{i=1}^{n} I_{i}^{v}},
\end{gathered}
$$

and obviously,

$$
\begin{aligned}
& p_{\text {mod }}=\sqrt{p_{x \text { mod }}^{2}+p_{y \text { mod }}^{2}}, \\
& Q_{0 \text { mod }}=\frac{1}{2} \arctan \left(\frac{p_{x \text { mod }}}{p_{y \text { mod }}}\right) .
\end{aligned}
$$

\section{References}

Angel, J. R. P., \& Stockman, H. S. 1980, ARA\&A, 18, 321

Gabuzda, D. C., et al. 2006, MNRAS, 369, 1569

Goldberg, D. E. 1989, Genetic Algorithms in Search, Optimization, and Machine Learning (Addison-Wesley, Massachusetts)

Hagen-Thorn, V. A. 1980, Ap\&SS, 73, 263

Hagen-Thorn, V. A., Larionova, E. G., Jorstad, S. G., et al. 2002, A\&A, 385, 55 (Paper I)

Jorstad, S. G., Marscher, A. P., Stevens, J. A., et al. 2007, AJ, 134, 799

Lister, M. L., \& Smith, P. S. 2000, ApJ, 541, 66

Marscher, A. P., Jorstad, S. G., D'Arcangelo, F. D., et al. 2008, Nature, 452, 966 McKinney, J. C. 2006, MNRAS, 368, 1561

Mead, A. R. G., Ballard, K. R., Brand, P. W. J. L., et al. 1990, A\&AS, 83, 183

Vlahakis, N., \& Königl, A. 2004, ApJ, 605, 656 